\title{
Depresión postparto en población inmigrante en Santiago: estudio transversal y descriptivo
}

DOI: $10.46981 /$ sfjhv2n2-015

Received in: April 1st, 2021

Accepted in: May 31th, 2021

\author{
Marcelo Tampier Jara \\ Médico Cirujano Universidad de los Andes, Santiago, Chile \\ Institución actual: Centro Comunitario de Salud Mental, Lo Barnechea \\ Dirección: Robles 13730, Lo Barnechea, Santiago, Chile \\ E-mail: marcelotampier@gmail.com
}

Giordanno Nobizelli Aguilar

Médico Cirujano Universidad de Los Andes, Santiago de Chile. Diplomado Medicina de Urgencias, Pontificia Universidad Católica de Chile.

Institución actual: Hospital San Pablo de Coquimbo

Dirección: Avenida Videla sin número, Coquimbo, Chile.

E-mail: nobizellig@gmail.com

\section{María Jesús Salles Sapag}

Médico Cirujano Pontificia Universidad Católica de Chile. Médico Psiquiatra Universidad de los Andes. Diplomado en Docencia en Carreras de la Salud, U. De los Andes. Institución actual: Docente Escuela de Psicología Universidad de Los Andes Dirección: Monseñor Álvaro del Portillo 12455, Las Condes, Santiago, Chile E-mail: mjsalles@uc.cl

\section{Matías Jorge Villagrán Salas}

Título: Médico Cirujano Universidad de los Andes, Santiago, Chile Institución actual: Hospital El Carmen, Maipú

Dirección: Camino a Rinconada 1201, Maipú, Santiago, Chile.

E-mail: mjvillagran@miuandes.cl

\section{Sofía Adela Gamboa Kornberg}

Título: Médico Cirujano Universidad de Los Andes, Santiago, Chile. Institución actual: Hospital Luis Tisné Brousse

Dirección: Avenida Las Torres 5150, Peñalolén, Santiago, Chile

E-mail: sagamboa@miuandes.cl

\section{Sebastián Cristobal Molina Osses}

Título: Médico Cirujano Universidad de los Andes, Santiago, Chile

Institución actual: CESFAM Michel Chandía Alarcón, Coihueco

Dirección: Camino a Huape km 11, Chillán, Chile.

E-mail: scmolina@miuandes.cl

\section{RESUMEN}

Introducción: La situación de inmigración puede generar cambios emocionales profundos producto de lo que implica llegar a un nuevo país; el impacto cultural, el idioma, la disminución de redes de apoyo e incluso la discriminación. La Depresión Postparto (DPP) es un trastorno psiquiátrico que se extiende según diversas definiciones- hasta 6 meses luego del parto, y su inicio y severidad es producido por 
numerosos factores, dentro de los cuales podría encontrarse la inmigración. Objetivo: Realizar un estudio exploratorio a mujeres inmigrantes de Chile, pesquisar su riesgo de tener DPP de acuerdo a la Escala de Depresión Postparto de Edimburgo y relacionar esto según las variables sociodemográficas recopiladas durante la entrevista. Metodología: la muestra incluyó 19 mujeres inmigrantes, con hijos entre 2 a 6 meses de edad nacidos en Chile, a quienes se le realizó una encuesta que abarcaba tanto datos personales; nacionalidad, convivencia con pareja, ocupación, partos previos y percepción de acogida, entre otros, como también la Escala de Edimburgo. Para esto, se entrevistaron personas en la vía pública en sectores de Estación Central, Santiago que cumplieran con los criterios de inclusión. Resultados: De las 19 mujeres encuestadas, 5 de ellas resultaron con $\geq 10$ puntos en la Escala de Edimburgo, correspondiendo a un $26,3 \%$ del total de la muestra, lo que significa que están en riesgo de padecer DPP. Conclusiones: No se logró identificar una relación estadísticamente significativa entre el riesgo de desarrollar DPP y el ser inmigrante, ni entre la relación de riesgo de DPP con las variables sociodemográficas analizadas. Se requieren más estudios para evaluar esta hipótesis.

Palabras claves: depresión post parto, inmigración, escala de Edimburgo, salud mental

\begin{abstract}
Introduction: Immigration to a new country involves many changes in people's lives as well as in their emotional well-being, most importantly related to language difficulties, loss of emotional support such as family and friends and in some cases discrimination. Postpartum Depression (PPD) is a relatively frequent psychiatric disorder found in women that lasts up to 6 months after giving birth and its onset and severity may be associated with many risk factors, including immigration. Objectives: to carry out an exploratory study of immigrant women in Santiago, Chile and investigate the risk of having PPD according to the Edinburgh PPD scale and to analyze its association with different sociodemographic variables obtained during the interview. Methodology: the sample consisted of 19 immigrant women with children of 2 to 6 months of age born in Chile who underwent an in-person survey covering both personal data, including; nationality, relationship status, occupation, family composition, numbers of births and abroad experience and finally the Edinburgh Postnatal Depression Scale Test. The sample was taken after meeting the inclusion criteria in people found in public spaces close to Metro Estación Central, Santiago. Results: of the 19 women who met the criteria, 5 of them reported a score of $\geq 10$ on the Edinburgh scale, which translates to $26.3 \%$ of the total sample. Conclusions: There was no statistical significance relating postnatal depression and inmigration and no correlation between risk of PPD and the sociodemographic variables analyzed. More studies are needed to verify this hypothesis.
\end{abstract}

Keywords: postpartum depression, immigration, Edinburgh postnatal depression scale.

\title{
1 INTRODUCCIÓN
}

En la actualidad, la inmigración ha sido un tema que ha ido cobrando más relevancia a nivel de Salud Pública. Los nuevos allegados traen consigo una carga social, cultural y de salud muy distinta a la realidad nacional. En el año 2014 se estimaron 411.000 inmigrantes permanentes en nuestro país, equivalentes al 2,3\% de la población total.

La condición de extranjería en Chile se define en base al tipo de Visa que estas personas tienen (Trabajo, Turismo y Permanencia). Lamentablemente, no existen muchos catastros que dan cuenta de la situación de los extranjeros, especialmente sobre aquellos que viven en condiciones más vulnerables. 
En relación a esto, la encuesta CASEN realizada el 2016, revela que en Chile un 33\% de los extranjeros vive en situación de pobreza (1). Otro estudio financiado por el BID (Banco Internacional del Desarrollo) y FOMIN (fondo multilateral de inversiones), mediante encuestas telefónicas y presenciales, encontró que un $44 \%$ de los extranjeros encuestados no cuentan con un sistema de previsión de salud, un 35\% se encuentra en situación de desempleo y un 9\% con una situación laboral inestable (2).

A lo anterior, varios estudios han descrito que la situación de inmigración puede generar episodios depresivos, producto del duelo que implica el cambio de país, como todo lo que significa adaptarse a un nuevo medio, asociándose esta condición a la ausencia de redes de apoyo sostenibles.

Por otro lado, cabe destacar que la OMS dentro de sus últimos estudios reveló que Chile presenta la mayor carga de morbilidad por enfermedad psiquiátrica del mundo con un 23,3\% (3), explicado en gran parte por las condiciones de desigualdad, acceso a salud y adherencia tratamientos ofrecidos. Particularmente, en la maternidad se han descrito diversos cuadros afectivos asociados que resultan de gran impacto para el desarrollo del apego madre-hijo y la conformación de núcleo familiar estable y seguro (4). Dentro de este marco, una de las patologías que cobra gran relevancia, tanto por su prevalencia de un $36.7 \%$ en Chile (5), como por su efectivo tratamiento, es la depresión post Parto (DPP). Actualmente en Chile no existen trabajos que vinculen la situación de inmigración con cuadros depresivos maternos.

La depresión post parto es un cuadro común, invalidante y tratable que afecta a mujeres, y secundariamente a niños y familias. De acuerdo a la DSM V, es un episodio depresivo de inicio perinatal o hasta 12 meses post parto, asociado a síntomas tales como trastornos del sueño no asociados a cuidados del bebé, ansiedad, irritabilidad, labilidad emocional, sentirse sobrepasado o con ideas obsesivas sobre la salud del hijo (6).

Tiene una prevalencia mundial que fluctúa de 10-20\%, siendo mayor en ciertos grupos de riesgo como adolescentes y condiciones de vulnerabilidad social. En el caso chileno, según estudios realizados, la prevalencia alcanza un 10,2-20,5\% con una incidencia del 8,8\% (4).

Esta patología presenta asociado además ciertos factores de riesgo descritos por la literatura, dentro de los cuales destacan: escasa red de apoyo, antecedentes de depresión, eventos vitales estresantes y problemas de pareja.

Por esto mismo, se realiza un screening en diversos momentos con la Escala de depresión Post Parto de Edimburgo (EDPNE), validada por el ministerio de Salud, y recomendada por la "American College of Obstetricians and Gynecologist" y la "American Academy of Pediatrics". Se indica su aplicación durante el primer o segundo control gestacional y en el post parto a las ocho semanas y al 
6to mes. Con 10 o más puntos, de un total de 30, se considera a la madre en riesgo de padecer depresión post parto.(6)

\section{MÉTODO}

\subsection{SELECCIÓN Y DESCRIPCIÓN DE LOS PARTICIPANTES}

La población estudiada fueron mujeres extranjeras residentes en Chile desde el año 2014, que hayan tenido su periodo de gestación completa en territorio nacional y que tengan un lactante entre 26 meses de vida, a través de un muestreo aleatorio no probabilístico. El punto de corte se fijó en el año 2014, dado que en ese año se constituyó en el MINSAL el Equipo Asesor Sectorial de Salud de Inmigrantes cuyo objetivo fue el desarrollo de la Política de Salud de Inmigrantes. A partir de ese trabajo se realizó una revisión de la normativa, donde destaca el Decreto $N^{\circ} 67$ que incorpora a los inmigrantes en situación irregular ( $\sin$ visa ni documentos), como beneficiarios de FONASA. Asimismo, se consideró relevante que las mujeres en estudio hayan tenido su gestación completa en Chile, puesto que el proceso de inmigración para una embarazada corresponde a un factor estresante que podría eventualmente desencadenar problemas psicológicos y por ende una mayor tendencia al desarrollo de depresión post parto.

Finalmente fue necesario solo incluir a madres con hijos entre 2-6 meses de vida porque son criterios necesarios para aplicar de forma fidedigna la "Escala de Depresión Post natal de Edimburgo" (EDPNE). Junto con ello, para generar una muestra más representativa, se excluyeron del estudio a mujeres con diagnóstico de enfermedad psiquiátrica en tratamiento.

\subsection{INFORMACIÓN TÉCNICA}

Dentro de los meses de agosto y septiembre de 2017, se realizaron encuestas presenciales a mujeres que cumplieran con los criterios de inclusión. Primeramente se les dio entrega de un consentimiento informado, previamente aprobado por Escuela de Medicina de la Universidad de los Andes, dejando a la población en libertad de participación. Junto con ello, debieron rellenar un formulario de datos personales y sociodemográficos considerando las variables de: edad, estado civil, previsión, ocupación, antecedente de embarazos previos, comorbilidades, cohabitantes y percepción de acogida chilena.

La segunda etapa de la entrevista, correspondió a la aplicación de la "Escala de Depresión Post Natal de Edimburgo" en las mujeres estudiadas, la cual es utilizada como screening para establecer sospecha de depresión post parto, validada a nivel internacional mediante estudio "Validation of the Edimburg postnatal depression scale in Chilean postpartum women" (2009), arrojando una sensibilidad de $100 \%$, especificidad de $80 \%$ y VPP de $31 \%$. La escala corresponde a un cuestionario auto realizable, 
compuesta por 10 ítems, cada uno de los cuales posee 4 opciones de respuestas calificadas como A-BC-D, de acuerdo a severidad de síntomas depresivos, siendo A "nunca" y D "siempre o mayor parte del tiempo". A partir de los resultados a las pacientes que marcaron $\geq 10$ puntos (sospecha de depresión post parto, por tanto EDPNE +), se les recomendó evaluación médica. Junto con ello, cualquier respuesta distinta de "A" en el ítem 10 (ideas de muerte), se les indicó evaluación médica dentro de un plazo de $24 \mathrm{~h}$.

\subsection{ANÁLISIS DE DATOS}

El resultado final generó un total de 19 mujeres entrevistadas, las cuales fueron ingresadas a una base de datos realizada en plataforma Excel. Se procedió clasificar a cada persona según el puntaje obtenido en la "Escala de Depresión Post Natal de Edimburgo" (EDPNE), denominándolas como personas "en riesgo" (EDPNE +) si es que obtuvieron puntaje $\geq 10$ puntos, o "sin riesgo" (EDPNE -) en aquellas que obtuvieron $<10$ puntos.

Posteriormente, se clasificó a la población en estudio según nacionalidad, situación laboral, antecedente de embarazo previo, previsión médica, personas con quien cohabita y percepción de acogida, y así determinar si la variabilidad de estos factores son eventualmente predisponentes a padecer la patología.

\section{RESULTADOS}

\subsection{RESULTADOS Y VARIABLES SOCIODEMOGRÁFICAS}

Tabla 1: Resultados de aplicación de la Escala de Edimburgo en el total de las madres encuestadas.

\begin{tabular}{|c|c|c|}
\hline \multicolumn{2}{|c|}{ Resultado EDPNE $N^{\circ}$ Total } & $\%$ del Total \\
\hline$(-)<10$ pts & 14 & $73.7 \%$ \\
\hline$(+) \geq 10$ pts & 5 & $26.3 \%$ \\
\hline
\end{tabular}

Puntaje promedio corresponde a 5.1 puntos. 
Tabla 2: Relación de riesgo entre pesquisa de DPP vs convivencia.

\begin{tabular}{|c|c|c|c|}
\hline Convivencia & $\mathbf{N}^{\circ}$ Total & $\begin{array}{c}\text { \% EDPNE (+)/N } \\
\text { Total }\end{array}$ & OR (IC 95\%) \\
\hline Sin pareja & 3 & $66 \%$ & $8.67(0.58-130)$ \\
\hline Con pareja & 16 & $18.8 \%$ & \\
\hline
\end{tabular}

Tabla 3: Relación de riesgo de pesquisa de DPP vs embarazo previo.

\begin{tabular}{c|c:c:c}
$\begin{array}{c}\text { Embarazos } \\
\text { previos }\end{array}$ & $N^{\circ}$ Total & $\begin{array}{c}\% \text { EDPNE }(+) / N^{\circ} \\
\text { Total }\end{array}$ & OR \\
\hline Ninguno & 12 & $25 \%$ & $0.83(0.1-6.8)$ \\
\hline Uno o más & 7 & $28.5 \%$ & \\
\hline
\end{tabular}

Tabla 4: Relación de riesgo de pesquisa de DPP vs actividad laboral.

\begin{tabular}{c|c:c:c} 
Trabajo & $\mathbf{N}^{\circ}$ Total & $\begin{array}{c}\text { \% EDPNE }(+) / \mathbf{N}^{\circ} \\
\text { Total }\end{array}$ & OR \\
\hline Fuera de casa & 6 & $33 \%$ & $1.66(0.2-14.05)$ \\
\hline En casa & 13 & $30 \%$ & \\
\hline
\end{tabular}

Tabla 5: Percepción subjetiva de acogida de Chile por parte de las madres.

\begin{tabular}{|c|c:c|c|}
\hline Acogida & $\mathbf{N}^{\circ}$ Total & $\begin{array}{c}\% \text { EDPNE }(+) / N^{\circ} \\
\text { Total }\end{array}$ & OR \\
\hline Mala & 1 & $0 \%$ & $0.92(0.07-11.6)$ \\
\hline Regular & 3 & $33 \%$ & \\
\hline Buena & 12 & $33 \%$ & \\
\hline Muy buena & 3 & $0 \%$ & \\
\hline
\end{tabular}

\section{DISCUSIÓN}

Si bien el hecho de ser extranjero en un país podría ser una condición que influya en el desarrollo de la DPP, en la población migrante se reúnen muchos otros factores de riesgo que por sí solos facilitan esta patología, por lo que sería importante indagar más en las condiciones socio-demográficas, especialmente en el caso de las mujeres embarazadas y en puerperio.

Dentro del análisis de los ítems por separado, cabe destacar que ninguna de las entrevistadas contestó afirmativo a la pregunta que evalúa riesgo suicida, que eventualmente podría haber requerido evaluación médica de urgencia

Por otro lado, dentro del contexto socio-demográfico se vio que: 
En la variable laboral, si bien no se puede aseverar la relación entre la situación de cesantía y una mayor o menor prevalencia de DPP, es importante recalcar el alto volumen de madres inmigrantes que no tienen una situación laboral estable, que de por sí genera una situación estresante y de incertidumbre en ellas.

En relación al Embarazo previo, donde se compararon las mujeres primigestas v/s las multíparas, el segundo grupo tenía levemente mayor riesgo de desarrollar depresión post parto, sin embargo, estadísticamente no es significativo (OR 0,83 (IC 95\%, 0,1-6,8).

En relación a la variable de persona con quien cohabita, ya sea pareja o esposo, se detectó que aquellas mujeres que convivían con su pareja, tenían menos probabilidad de desarrollar síntomas depresivos en el puerperio, a diferencia de las que no convivían con su pareja (18.8\% contra 66\% de EDPNE +). De acuerdo a esto, se podría deducir que hay una relación entre la convivencia con la pareja y el riesgo de desarrollar DPP, donde el hecho de tener una pareja podría ser una situación de apoyo en el embarazo que prevendría el riesgo del desarrollo de DPP (OR = 8.67; IC 95\% 0.58-130), coincidiendo con lo planteado en las guías clínicas del MINSAL, en donde se establece "no vivir con la pareja" como un factor de riesgo moderado, o "yellow flag" para el desarrollo de DPP. Por lo anterior, sería algo importante a considerar al momento de la acogida de las madres extranjeras en Chile con medidas de respaldo emocional y psicológico a nivel nacional y de salud pública.

Asimismo, con respecto a la percepción que tienen las mujeres estudiadas con respecto a la acogida en Chile, destacó un 79\% (n=15) de inmigrantes que tenían una buena percepción de la acogida en Chile, un $16 \%(n=3)$ de regular percepción y 5.2\% $(n=1)$ de mala percepción. Además, según los datos de la tabla, los porcentajes se acercan al promedio excepto en el caso de 1 encuestada con mala percepción, lo que es un N muy bajo como para sugerir un factor protector a DPP (+) en relación a la mala percepción. De acuerdo a esto, si bien no se encontró una relación evidente entre la percepción de la acogida en Chile y el riesgo de padecer DPP, destaca la opinión de los inmigrantes acerca de su acogida en el país, el cual tiende a ser más bien positivo.

Respecto a las limitaciones que se tuvieron en el estudio, en primera instancia mencionar los estrictos criterios de inclusión establecidos por la investigación que acotaron bastante la muestra.

Asimismo, otra limitante fue el idioma, puesto que gran parte de la población inmigrante a la cual pudimos acceder, correspondía a haitianos no hispano hablantes. Por este motivo, no se pudo aplicar la encuesta, ya que no está validada en su lengua nativa y por ende disminuyó notoriamente el tamaño de la muestra y por tanto la representación de la población inmigrante en Chile de aquel momento.

En cuanto a los posibles aspectos a mejorar en un futuro trabajo de investigación de esta misma materia, destaca principalmente el ampliar el tamaño de la población estudiada modificando los 
métodos de abordaje y tamizaje, generando así una muestra más representativa y de la cual realizar análisis más contundentes, así como también evaluar la generación de instrumentos de evaluación validados en otros idiomas para disminuir al máximo los sesgos de selección.

\section{RECOMENDACIONES}

A pesar de que se encontró un menor porcentaje de mujeres inmigrantes en riesgo de depresión postparto, en relación a la prevalencia nacional de la misma patología, se hace imprescindible que tanto a nivel gubernamental como social generemos un ambiente de solidaridad frente a estas mujeres, que día a día suman en número sobretodo en nuestra capital.

Como ciudadanos tenemos la misión de hacer de Chile un país inclusivo y solidario, evitando los típicos prejuicios y estigmas.

Por esto, se invita a otras personas interesada en explorar el tema, a hacer análisis basados en modelos estadísticos más sofisticados para lograr comprender con mayor cabalidad cuales son las verdaderas necesidades de la población inmigrante y poder desarrollar así soluciones y políticas más afines. 


\section{REFERENCIAS}

(1) Ministerio de Desarrollo Social, División Social, Encuesta CASEN www.ministeriodesarrollosocial.gob.cl/observatorio/casen/

(2) Foreign workers, Latin American-Chile. 2. Emigrant remittances-Chile. 3. Saving and investmentChile. 5. Financial services industry-Chile. 6. ChileEmigration and inmigration-Economic aspects. 7. Chile-Emigration and inmigration-Social aspects. I. Tessada, José. II. Banco Interamericano de Desarrollo. Oficina del Fondo Multilateral de Inversiones.

(3) Vicente Benjamín, Saldivia Sandra, Pihán Rolando. Prevalencias y brechas hoy: salud mental mañana. Acta bioeth. [Internet]. 2016 Jun [citado 2017 Jun 14] ; 22( 1 ): 51-61. Disponible en: http://www.scielo.cl/scielo.php?script=sci_arttext\&pid=S1726- $\quad$ 569X2016000100006\&lng=es. http://dx.doi.org/10.4067/S1726-569X2016000100006.

(4) Mendoza B Constanza, Saldivia Sandra. Actualización en depresión postparto: el desafío permanente de optimizar su detección y abordaje. Rev. méd. Chile [Internet]. 2015 Jul [citado 2017 Jun 14] ; 143( 7 ): 887-894. Disponible en: http://www.scielo.cl/scielo.php?script=sci_arttext\&pid=S003498872015000700010\&lng=es. http://dx.doi.org/10.4067/S0034- 98872015000700010.

(5) Jadresic E, Araya R. Prevalencia de depresión posparto y factores asociados en Santiago, Chile. RevMéd Chile 1995; 123: 694-9

(6) Stewart DE, Vigod S. Postpartum Depression. Solomon CG, editor. New England Journal of Medicine. diciembre de 2016;375(22):2177-86. 